\title{
Validation of computed extreme ultraviolet emission spectra during solar flares
}

\author{
Shohei Nishimoto ${ }^{1 *}$, Kyoko Watanabe ${ }^{1}$, Toshiki Kawai ${ }^{2}$, Shinsuke Imada ${ }^{2}$ and Tomoko Kawate ${ }^{3}$
}

\begin{abstract}
X-rays and extreme ultraviolet (EUV) emissions from solar flares rapidly change the physical composition of the Earth's thermosphere and ionosphere, thereby causing space weather phenomena such as communication failures. Numerous empirical and physical models have been developed to estimate the effects of flare emissions on the Earth's upper atmosphere. We verified the reproduction of the flare emission spectra using a one-dimensional hydrodynamic calculation and the CHIANTI atomic database. To validate the proposed model, we used the observed EUV spectra obtained by the Extreme ultraviolet variability experiment (EVE) on board the Solar Dynamics Observatory (SDO). We examined the "EUV flare time-integrated irradiance" and "EUV flare line rise time" of the EUV emissions for 21 events by comparing the calculation results of the proposed model and observed EUV spectral data. The proposed model successfully reproduced the EUV flare time-integrated irradiance of the Fe VIII $131 \AA$, Fe XVIII $94 \AA$, and Fe XX133 $\AA$, as well as the 55-355 $\AA$ and 55-135 $\AA$ bands. For the EUV flare line rise time, there was an acceptable correlation between the proposed model estimations and observations for all Fe flare emission lines. These results demonstrate that the proposed model can reproduce the EUV flare emission spectra from the emitting plasma with a relatively high formation temperature. This indicates that the physics-based model is effective for the accurate reproduction of the EUV spectral irradiance.
\end{abstract}

Keywords: Solar flare, X-ray emission, EUV emission, Space weather

\section{Introduction}

To consider outer space as a place for deploying advanced space activities, a common fundamental system, called space infrastructure, is required. To build and use a space infrastructure safely, it is important to accurately understand the current state of the solar-terrestrial environment, which is strongly influenced by solar activity. Among the solar activity, solar flares have one of the greatest influences on the solar-terrestrial environment. When solar flares occur, powerful electromagnetic radiation and large amounts of high-energy particles are released. Among these, it is well known that X-ray and extreme ultraviolet (EUV) emissions of solar flares, in

\footnotetext{
${ }^{*}$ Correspondence: ed19004@nda.ac.jp

1 National Defense Academy of Japan, 1-10-20 Hashirimizu,

Yokosuka 239-8686, Japan

Full list of author information is available at the end of the article
}

particular, influence the Earth's communication network. When X-ray and EUV emissions from solar flares reach the Earth's upper atmosphere, especially the D layer of the ionosphere, oxygen and nitrogen in the D layer are ionized. Consequently, the electron density in the D layer increases rapidly, and radio waves (especially at high-frequency ranges) propagating through the D layer are absorbed. This phenomenon is called the Dellinger phenomenon (Dellinger 1937), which is widely known as a sudden ionospheric disturbances (SIDs). Solar flare emissions reach the Earth in $\sim 8 \mathrm{~min}$, and the lead time from solar flares to SIDs is extremely short. Therefore, it is important for the solar irradiance, especially X-ray and EUV emissions, to be constantly monitored.

In general, SIDs are considered to be caused by the occurrence of solar flares of the M-class or higher and can be predicted using the flare class. However, it has been reported that SIDs have occurred due to C-class 
flares but not due to X-class flares. These observational results suggest that flare emissions contributing to the occurrence of SIDs is not necessarily proportional to the $\mathrm{X}$-ray intensity. Observational data of the full solar flare emission spectrum are required to verify the influence of solar flare emission wavelengths on the occurrence of SIDs. However, spectral observations of EUV and X-ray emissions, which are considered to contribute significantly to SIDs, are limited.

$\mathrm{X}$-ray emissions have been continuously observed since 1974 using X-ray Sensors (XRS) on board the Geostationary Operational Environmental Satellite (GOES). GOES/XRS observes two wavelength bands, 0.5-4 $\AA$ (GOES/XRS-A) and 1-8 $\AA$ (GOES/XRS-B) (Bornmann et al. 1996).

EUV emissions have been observed using various instruments because of their importance in space weather forecasting. The Solar and Heliospheric Observatory $(\mathrm{SOHO})$ satellite, which was launched in December 1995, has a Solar EUV Monitor (SEM) (Judge et al. 1998). This instrument has been observing EUV emissions with a 15-s time resolution since January 1996. However, SOHO/SEM only has two wavelength bands,

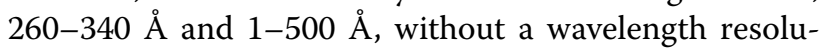
tion. Hence, only the temporal variation in these EUV emissions can be determined from the SOHO/SEM data. The Thermosphere Ionosphere Mesosphere Energetics and Dynamics (TIMED) satellite, which was launched in December 2001, has an EUV observation instrument called the Solar EUV Experiment (SEE) (Woods et al. 2005). TIMED/SEE has been observing the wavelength range of 1-1940 $\AA$ with a $4 \AA$ resolution since January 2002 and has superior spectral resolution compared to $\mathrm{SOHO} / \mathrm{SEM}$. However, its time resolution is $\sim 1$ day, and as hence, short-term fluctuations such as solar flares cannot be measured. The Extreme Ultraviolet Sensors (EUVS) onboard the GOES-R observes the eight EUV lines or bands in the wavelength range of $250-2850 \AA$ with a 10.24 s cadence since 2016 (Eparvier et al. 2009; Thiemann et al. 2019). The Solar Dynamics Observatory (SDO) satellite launched in February 2010 includes the Extreme Ultraviolet Variability Experiment (EVE) (Woods et al. 2012). The Multiple EUV Grating Spectrograph (MEGS), a subsystem of the SDO/EVE, has measured full disk solar irradiance in the 1-1060 Å range with $1 \AA$ spectral resolution and a $10 \mathrm{~s}$ time cadence since May 2010. MEGS-A observes a wavelength range of 50-370 ̊, while MEGS-B observes a wavelength range of 350-1050 $\AA$. MEGS-A was the only instrument that could make observations throughout the day with sufficient spectral and temporal resolution to study the EUV flare emission spectra; however, this device was terminated in May 2014 due to a charge-coupled device (CCD) power anomaly. Although MEGS-B remains in operation today, it is possible that some flares may not be observed because MEGS-B can only operate for $\sim 3 \mathrm{~h}$ a day. Therefore, the observation of high-resolution EUV emission spectra during the occurrence of a solar flare occur is not guaranteed.

To model the emission spectra, it is necessary to understand the emission mechanism in solar flares. The basic conceptual model for solar flares is the CSHKP model (Carmichael 1964; Sturrock 1966; Hirayama 1974; Kopp and Pneuman 1976; Yokoyama and Shibata 1998; Shiota et al. 2005). According to this model, solar flares are caused by "magnetic reconnection" generated in the solar corona (Innes et al. 2003; Imada et al. 2013; Warren et al. 2018). The strong magnetic tension generated by the magnetic reconnection accelerates the electrons or protons in the solar corona. The accelerated particles travel downward along the magnetic field lines, fall into the chromosphere, and rapidly heat the high-density plasma. High-temperature and high-density plasma rise from the chromosphere along the magnetic field lines and form a loop-shaped structure; this phenomenon is called "chromospheric evaporation" (Milligan and Dennis 2009; Imada et al. 2015; Lee et al. 2017). The loop structure observed by soft X-ray and EUV formed from chromospheric evaporation is called the "flare ribbon". Soft X-ray and EUV emissions are emitted from the flare loop. The time evaporation of the EUV emissions is characterized by the temperature of the emitting plasmas. A typical flare light curve of an EUV emission has an impulsive peak initially, followed by a gradual peak, called the impulsive and gradual phases, respectively. In general, relatively cooler EUV line emissions from the plasma below the transition region are observed in the impulsive phase corresponding to the rapid heating in the early stages of chromospheric evaporation. Hotter EUV line emissions are observed in the gradual phase corresponding to the radiative cooling of the flare loop. Therefore, there are differences between the emission spectra of the impulsive and gradual phases because their origins are different.

Different flare EUV emission prediction models were constructed based on the above flare emission mechanism. The most widely used model is the Flare Emission Spectral Model (FISM) (Chamberlin et al. 2006, 2007, 2008). The FISM is an empirical model that derives EUV emission spectra using GOES soft X-ray flux observations. The FISM estimates the wavelength range of $1-1900 \AA$ with a $10 \AA$ spectral resolution and $60 \mathrm{~s}$ cadence. It has been reported that the FISM can accurately estimate the solar flare emission spectra within $40 \%$ for wavelengths in the range of 140-1900 A. However, FISM has a low accuracy for wavelengths shorter 
than $140 \AA$; this is the wavelength range containing the EUV emission lines that are primarily enhanced during the gradual phase of a flare. This is because the FISM considers the time evolution of all EUV line emissions to be the same as the time evolution of the soft X-ray during the flare, and the cooling of the flare loop (time difference of EUV line emissions) is not well represented. Consequently, the FISM underpredicts the flare duration and deposited energy (Thiemann et al. 2017).

The $\mathrm{Q}_{\mathrm{EUV}}$, for which the 0-450 $\AA$ EUV band is an important indicator of the EUV irradiance input to the Earth's upper atmosphere (Strickland et al. 1995). The EUV irradiance in the 0-70 $\AA$ and 70-170 $\AA$ bands has the greatest contribution to the $\mathrm{Q}_{\mathrm{EUV}}$ (Woods et al. 2011). Therefore, the EUV irradiance at wavelengths shorter than $140 \AA$ is important but cannot be accurately estimated by the FISM. To solve this discrepancy, it is necessary to accurately estimate the time evolution of the EUV emissions.

Other physics-based models have been constructed for this purpose; however, the EUV emission was only partially reproduced ( $\mathrm{Li}$ et al. 2014; Zeng et al. 2014). Thiemann et al. (2017) partially succeeded in modeling the timing of coronal loop cooling using an empirical rule. These studies used a zero-dimensional hydrodynamic model to simulate the thermal evolution of the coronal loops, called the Enthalpy-based Thermal Evolution of Loops (EBTEL) model, which can calculate the temperature and density in the flare loop without calculating the spatial loop evolution (Klimchuk et al. 2008; Cargill et al. 2012). However, the EBTEL model did not calculate the spatial distribution of the emitting plasma in the flare loop; hence, it is possible that the emission from the transition region plasma would not be reproduced accurately (Kawai et al. 2020). Kawai et al. (2020) introduced a new method for reproducing EUV flare emission spectra by considering the time evolution of the plasma distribution in the flare loop. This method was constructed using a one-dimensional hydrodynamic calculation and an atomic database. The details of this method are described in Sects. 3 and 4.

In this paper, we present the statistical results of the EUV emission spectra observed by SDO/EVE for 21 flare events and compare these with the spectra reproduced by Kawai et al. (2020). The purpose of this study is to investigate the accuracy of reproducing solar flare emission spectra using a simple method based on the physics of flare loops and to examine the important parameters for reproducing solar flare emissions.

The remainder of this paper is organized as follows: Sect. 2 presents the extraction of the comparative parameters using GOES/XRS and SDO/EVE. Section 3 introduces the models used in this study. Section 4 describes an example of the derivation of the solar flare emission spectra using the proposed model. Section 5 presents the statistical results of the comparison between the proposed model simulations and observations and verifies the solar flare emission spectra. Section 6 discusses and summarizes the results of the comparison.

\section{Data}

\subsection{Soft X-ray observation}

We used soft X-ray data observed by the GOES/XRS. The flare class and duration are typically determined using the GOES/XRS-B light curve observations. In this study, the flare start, peak, and end times were defined using the time derivative data of the GOES/XRS-B light curve observations to retain the impulsive and gradual phases. Furthermore, we divided the flare duration into rise and decay times to comprehensively investigate the flare evolution. Figure 1 displays an example of determining the rise and decay times for the M9.9-class flare on January 1, 2014. The start time and rise time are determined when the positive derivative value is observed continuously in GOES/XRS-B to the peak time, where the start time is the first instance of this continuous positive derivative. The end time and decay time are determined when the negative derivative value is observed continuously in GOES/XRS-B from the peak time to the end time, where the end time is the last instance of this continuous negative derivative.

\subsection{EUV flare emission spectra observation}

We examined the EUV emission spectra obtained from the SDO/EVE MEGS-A observations. For this study, we selected flare events greater than the M3-class flare that occurred between November 2010 and May 2014 from the Hinode flare catalogue (Watanabe et al. 2012). We used 21 events observed by the SDO/EVE MEGS-A during this period.

Figure 2 displays the EUV spectrum at GOES/XRS-B defined flare peak time (18:52 UT) for the M9.9-class flare on January 1, 2014, as observed by the SDO/EVE MEGS-A. This EUV spectrum subtracted the nonenhanced, pre-flare spectrum (18:00 UT) before the flare as background to obtain a flare-only spectrum, which is a common practice for analyzing flare emissions using emission spectra. We focused on the six EUV flare lines of Fe VIII $131 \AA$, Fe XV $284 \AA$, Fe XVI $335 \AA$, Fe XVIII 94 $\AA$, Fe XX $133 \AA$, and He II $304 \AA$, which were strongly enhanced during the flare. The formation temperature of the Fe XX $133 \AA$ is $\sim 10 \mathrm{MK}$, Fe XVIII $94 \AA$ is $\sim 6 \mathrm{MK}$, Fe XVI $335 \AA$ is $\sim 3$ MK, Fe XV $284 \AA$ is $\sim 2 \mathrm{MK}$, Fe VIII $131 \AA$ is $\sim 0.4 \mathrm{MK}$, and He II $304 \AA$ is $\sim 0.06$ MK (Woods et al. 2012). Figure 3 displays the EUV light curves of these six flare lines during the M9.9-class flare on January 


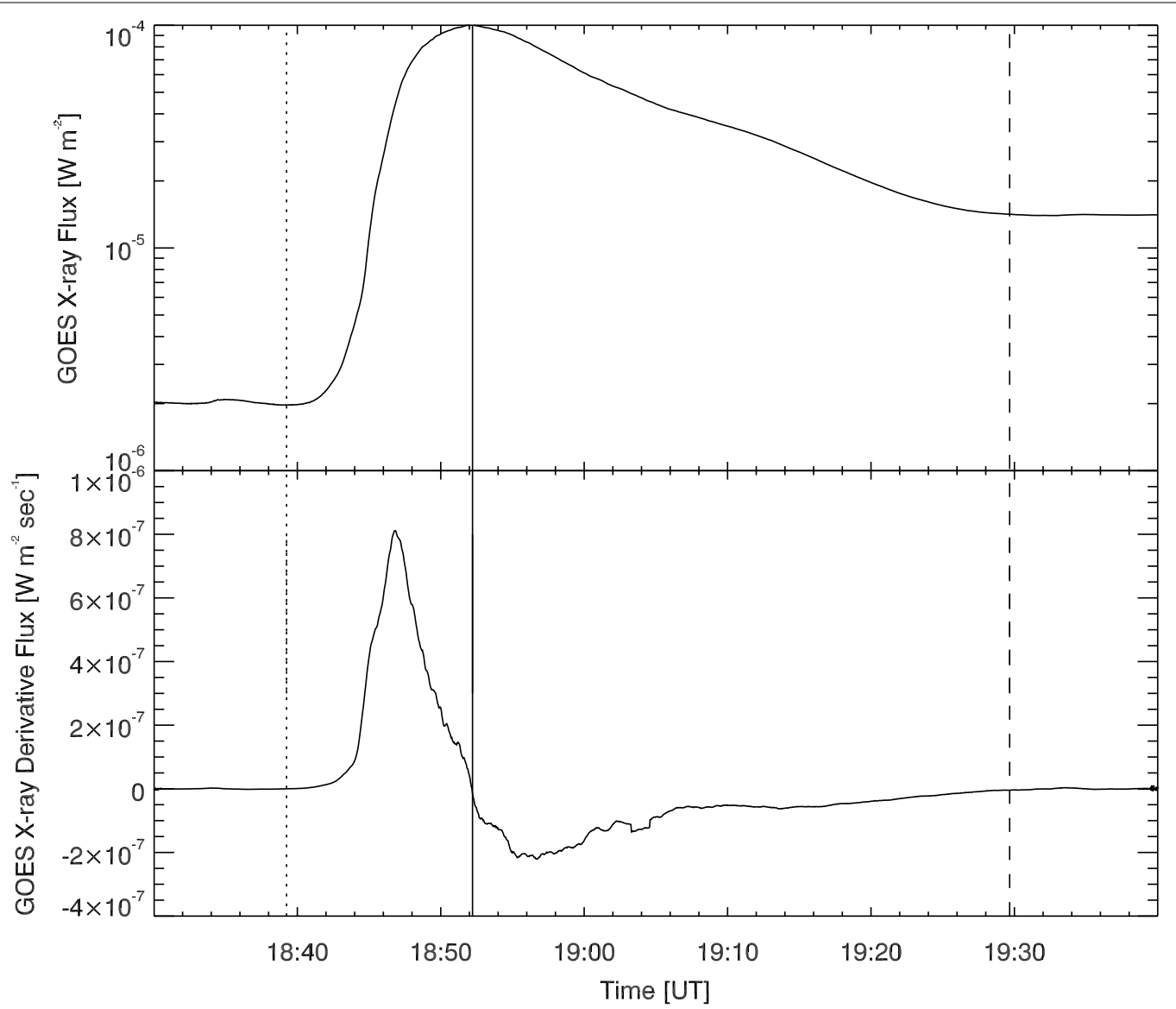

Fig. 1 Example of GOES/XRS-B observation. The light curve of soft X-ray flux (1-8 Å) observed by the GOES/XRS-B for the M9.9-class flare on January 1, 2014 (upper panel). Light curve of soft X-ray time derivative flux (lower panel). The vertical dotted, solid, and dashed lines indicate the flare start time, peak time, and end time, respectively

1, 2014. We subtracted a 3-min average non-enhanced, pre-flare value at the beginning of the hour before the flare start for each line. Furthermore, we used $110 \mathrm{~s}$ as the running average to remove noise due to short-term fluctuations (solid red line in Fig. 3).

To investigate the flare deposited energy and duration, which have a significant influence on the Earth's thermosphere and ionosphere response (Qian et al. 2011), we extracted the "EUV flare time-integrated irradiance" and the "EUV flare line rise time" as comparison parameters.

The EUV flare time-integrated irradiance is the timeintegrated value of the irradiance from the flare start to end time. We examined the EUV flare lines $55-355 \AA$ and 55-135 ̊̊ band. The wavelength band of 55-135 ̊ has a large uncertainty in the FISM. This wavelength range has a significant influence on the Earth's ionosphere (Woods et al. 2011; Thiemann et al. 2017). This relatively short wavelength band consists of hot EUV lines and dominates the EUV emissions in the gradual phase (Woods et al. 2011). The EUV flare rise time is the duration from the start of the flare to the EUV line peak time, as with the abovementioned soft X-ray observation.

\section{Models}

We reproduced the solar flare emission using a onedimensional hydrodynamic calculation and atomic database. First, we calculated the time evolution of the plasma in the flare loop, which is the source of X-rays and EUV during solar flares. We obtained the time variation of the X-rays and EUV emission spectra corresponding to the simulated emission plasma in the flare loop with the atomic database.

\subsection{Coordinated Astronomical Numerical Software (CANS) 1D package}

We used the one-dimensional hydrodynamic model called the CANS 1D package (http://www-space.eps.s.u-tokyo.ac. jp/ yokoyama/etc./cans/index-e.html) to solve the physical process of the plasma in the flare loop. This package can calculate the energy redistribution process in the flare loop 


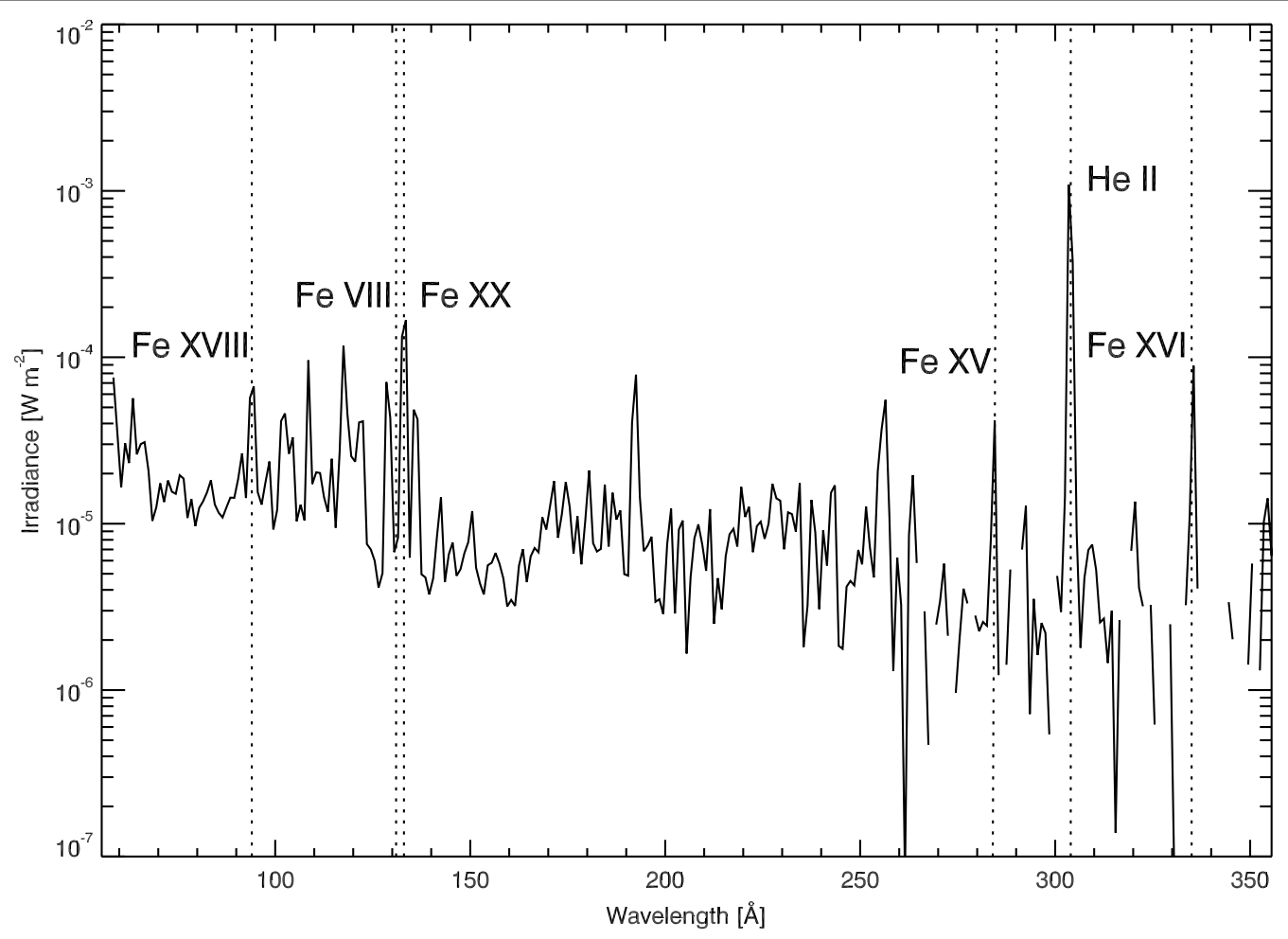

Fig. 2 Example of EUV spectrum observation. The EUV spectrum at the GOES/XRS-B peak time (18:52 UT) was observed by the SDO/EVE MEGS-A for the M9.9-class flare on January 1, 2014. This EUV spectrum is subtracted non-enhanced value (at 18:00 UT) from the value of each wavelength before the flare as the background. The EUV flare lines focused on in this study are indicated by vertical dotted lines

that occurs after the energy input. The simulation setup in this package is the same as that used by Hori et al. (1997) and Imada and Zweibel (2012). CANS 1D simulates onedimensional fluid motion and energy transfer along an invariant magnetic loop. It is assumed that the cross-section of the magnetic loop does not change over time. The fluid was considered as non-viscous and compressible and included heat conduction and radiative cooling. Gravity was also considered in this study. The fundamental equations of CANS 1D are as follows:

$$
\begin{aligned}
& \frac{\partial}{\partial t}(\rho S)+\frac{\partial}{\partial x}\left(\rho V_{x} S\right)=0, \\
& \frac{\partial}{\partial t}\left(\rho V_{x} S\right)+\frac{\partial}{\partial x}\left[\left(\rho V_{x}^{2}+p\right) S\right]=\rho g S,
\end{aligned}
$$

conductivity coefficient, $H$ is the static heating, $R$ is the radiative cooling, $H_{\mathrm{f}}$ is the flare heating term, $k_{\mathrm{B}}$ is the Boltzmann constant, and $m$ is the mean particle mass. The Spitzer model was used to calculate the thermal conductivity coefficient (Spitzer 1962). The calculation region is from the foot point of the flare loop, which includes the photosphere $(x=0)$ to the flare loop top $(x=L)$, where $L$ is the half-loop length.

Flare heating is given as a function of time and space, as follows:

$$
\begin{aligned}
& H_{\mathrm{f}}=H_{\mathrm{f} 0} \cdot q(t) \cdot f(x), \\
& q(t)=\frac{1}{4}\left\{1+\tan h \frac{t}{0.1 \tau_{0}}\right\}\left\{1-\tan h \frac{t-\tau_{\mathrm{f}}}{0.1 \tau_{0}}\right\},
\end{aligned}
$$

$$
\frac{\partial}{\partial t}\left[\left(\frac{p}{\gamma-1}+\frac{1}{2} \rho V_{x}^{2}\right) S\right]+\frac{\partial}{\partial x}\left[\left(\frac{\gamma}{\gamma-1} p+\frac{1}{2} \rho V_{x}^{2}\right) V_{x} S-\kappa \frac{\partial T}{\partial x} S\right]=\left(\rho g V_{x}+H-R+H_{\mathrm{f}}\right) S,
$$

$$
p=\frac{k_{\mathrm{B}}}{m} \rho T,
$$

where $\rho$ is the plasma density, $S$ is the cross-section, $V_{x}$

$$
f(x)=\frac{1}{\sqrt{2 \pi}} \exp \left[\frac{(x-L)^{2}}{2 w_{\mathrm{f}}^{2}}\right] \cdot \frac{1}{2}\left\{1+\tan h\left(\frac{x-20 \mathcal{H}_{0}}{3 \mathcal{H}_{0}}\right)\right\}
$$
tion, $\gamma=5 / 3$ is the specific heat ratio, $\kappa$ is the thermal 


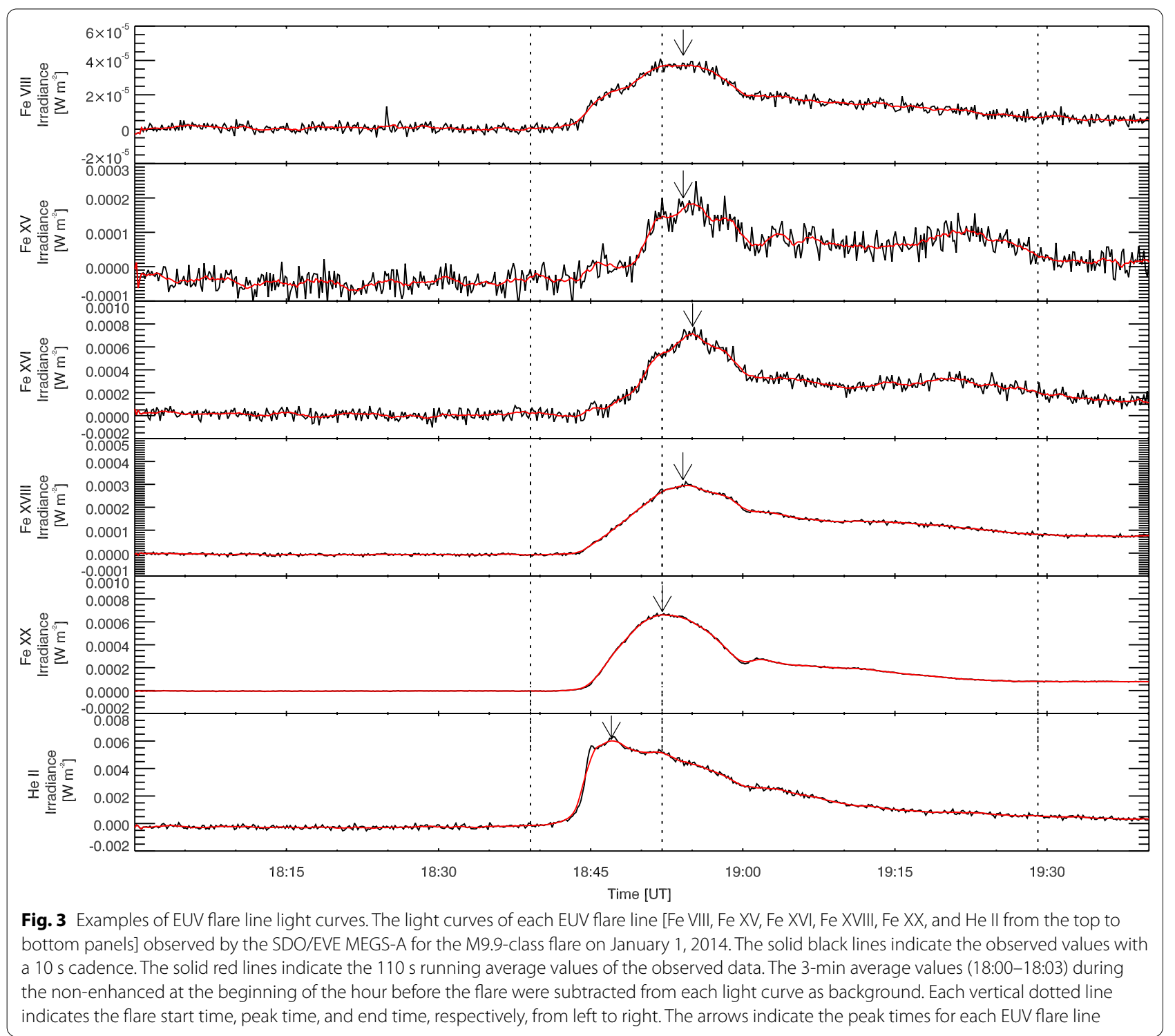

where $H_{\mathrm{f} 0}$ is the input energy, $\tau_{\mathrm{f}}$ is the heating duration, and $w_{\mathrm{f}}$ is the heating width. $\mathcal{H}_{0}$ is the scale height of the photosphere $(x=0)$.

\subsection{CHIANTI atomic database}

We used the CHIANTI atomic database to reproduce the X-ray and EUV emissions originating from the flare loop. The CHIANTI database contains a large amount of atomic data for the analysis of astrophysical spectra including atomic energy levels, wavelengths, radiative transition probabilities, rate coefficients for ionization, and data to calculate different emissions. In this study, we used the CHIANTI atomic database (version 9.0) (Dere et al. 1997, 2019).
4 Validation of M9.9-class flare on January 1, 2014 We compared the EUV flare time-integrated irradiance and EUV flare line rise time obtained from the model proposed by Kawai et al. (2020) and SDO/EVE MEGSA observations.

\subsection{Preprocessing}

To simplify the derivation of the EUV emission spectrum, the rise and decay times were fitted to exponential curves using the Gauss-Newton method [see Section 3.1, Kawai et al. (2020)]. Figure 4 displays an example of the fit GOES/XRS-B light curve for the M9.9-class flare on January 1, 2014. 


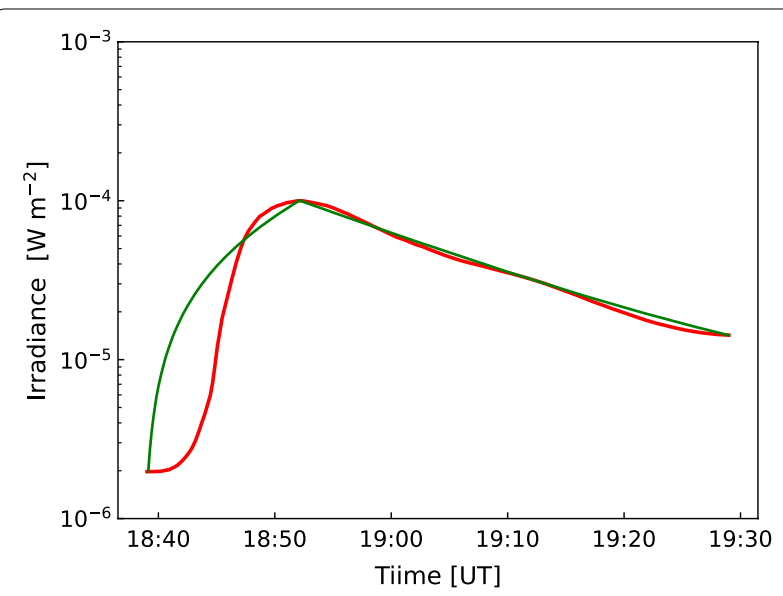

Fig. 4 Example of fit GOES/XRS-B light curve for the M9.9-class flare on January 1, 2014. The observed (red) and fit (green) light curves during the M9.9-class flare on January 1, 2014 using the GaussNewton method

\subsection{Coronal loop length measurement}

The flare loop length for the parameter used in CANS 1D was estimated by observing of the separation distance of the ribbons, called ribbon distance, with the SDO/Atmospheric Imaging Assembly (AIA) (Lemen et al. 2012). We used $1600 \AA$ images to obtain the ribbon distance immediately after the start of the flare. Before deriving the ribbon distance, we corrected the projection effect by rotating the flaring region to the solar center using the solar software drot_map.pro. First, we defined the flare ribbon as a region with intensities 40 times greater than the standard deviation of the quiet region. The ribbon distance is derived as the distance between the two brightest points in the flare ribbons and is known as the $H \alpha$ (UV) kernel (Asai et al. 2003; Temmer et al. 2007). For the M9.9-class flare on January 1, 2014, as indicated in Fig. 5, the ribbon distance was derived as 23.3 arcsec. Therefore, the ribbon distance of this event was estimated to be $16.9 \mathrm{Mm}$. Subsequently, the loop length was estimated to be $26.5 \mathrm{Mm}$ by assuming a semicircle whose diameter was this ribbon distance.

\subsection{Numerical simulation}

We reproduced the time evolution of the plasma in the flare loop with CANS 1D as described in Sect. 3.1. Figure 6 shows an example of the calculated results for CANS 1D. In this case, we set $L$ to $26.5 \mathrm{Mm}, H_{\mathrm{f} 0}$ was

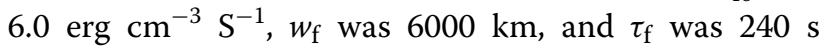
(Eqs. (5)-(7)). We determined $L$ from the observation of the ribbon distance described in Sect. 4.2, $H_{\mathrm{f} 0}$ was selected arbitrarily, and the magnitude of the computed GOES/XRS light curve are scaled to best match the

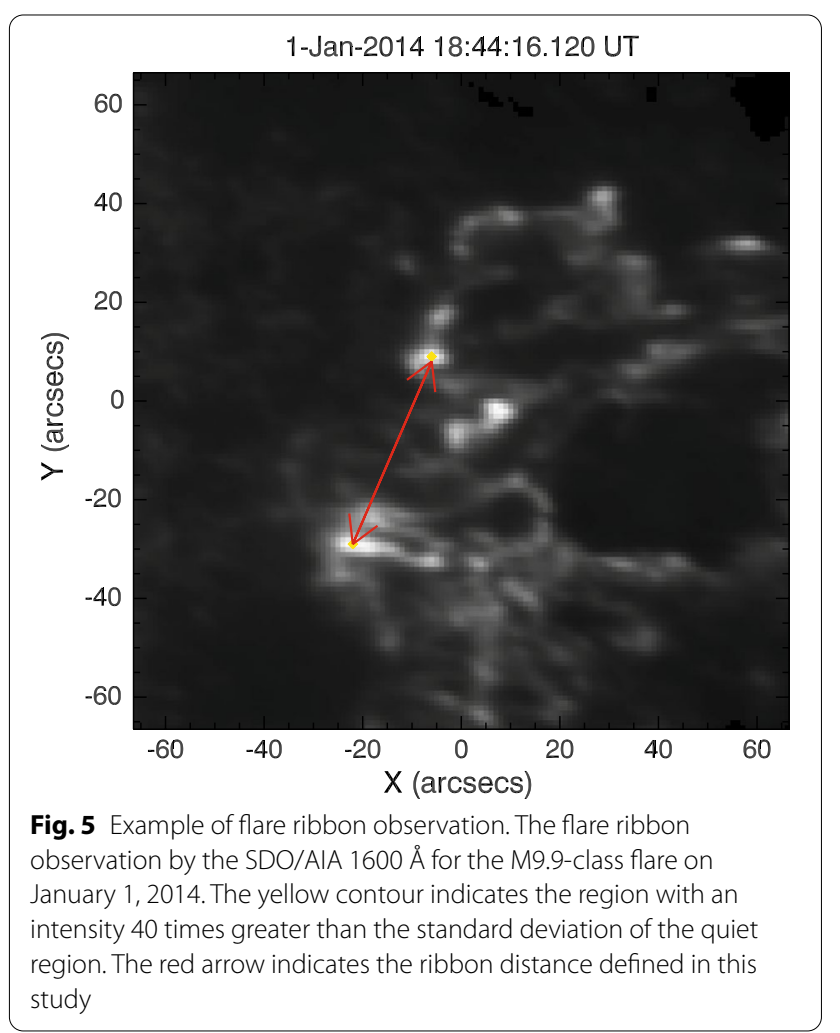

observation, $w_{\mathrm{f}}$ and $\tau_{\mathrm{f}}$ were from the default parameter values of CANS 1D (see the CANS 1D documentation). Although $w_{\mathrm{f}}$ and $\tau_{\mathrm{f}}$ should be different for each flare, the difference are difficult to observe; therefore, the typical flare values in CANS 1D were used as they were given. From the results, we identified the transition region to be near $0.3 \mathrm{Mm}$. In the figure, the temperature and density in the flare loop rise sharply in a couple of hundred seconds and at a height of $0.3 \mathrm{Mm}$, and the edge moves temporally. This represents the evolution of chromospheric evaporation. When flare occurs, accelerated particles fall on the chromosphere along the magnetic field lines. Consequently, the low-temperature plasma in the chromosphere is rapidly heated and transported upward owing to the pressure gradient. Hence, this calculation can reproduce the time evolution of plasma in the flare loop.

\subsection{Conversion from GOES X-ray to EUV spectrum}

We calculated the temporal variation and distribution of temperature, density, pressure, and plasma velocity of the emitting plasma in the single loop using CANS 1D (shown in Fig. 6). Then, we derived the synthetic emissions corresponding to the CANS 1D results using the CHIANTI atomic database (version 9.0) (Dere et al. 1997, 2019). The abundance file used in this study is "sun_coronal_2012_schmelz" (see Section 3.4, Kawai et al. 2020). 
a

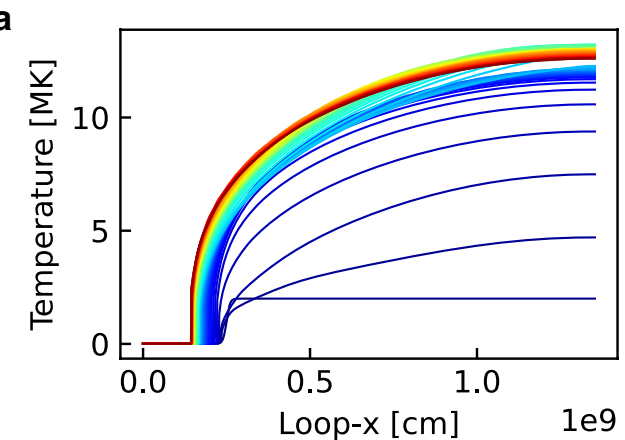

C

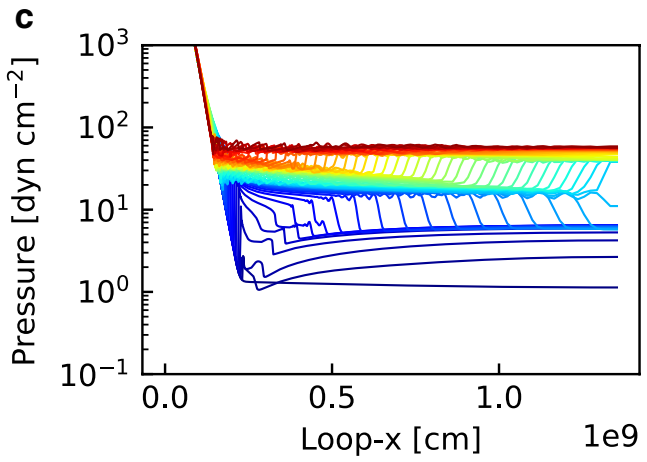

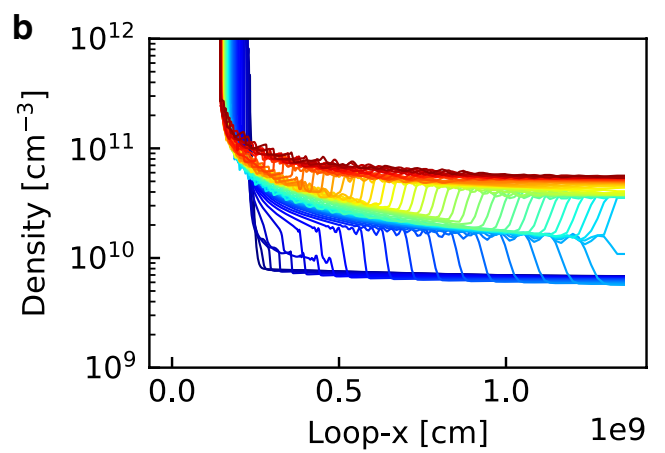

d

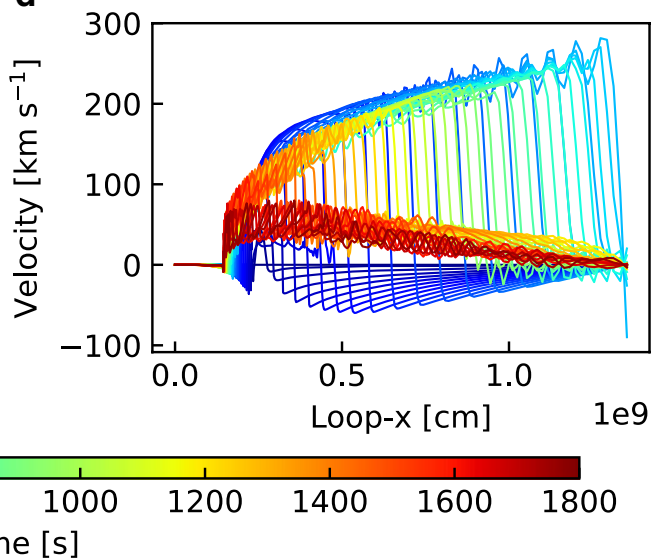

Fig. 6 Calculated results of CANS 1D. The hydrodynamic calculation results with CANS 1D for a temperature distribution, $\mathbf{b}$ density, $\mathbf{c}$ pressure, and d plasma velocity along the loop. The time evolution is represented by the change in line color from blue to red

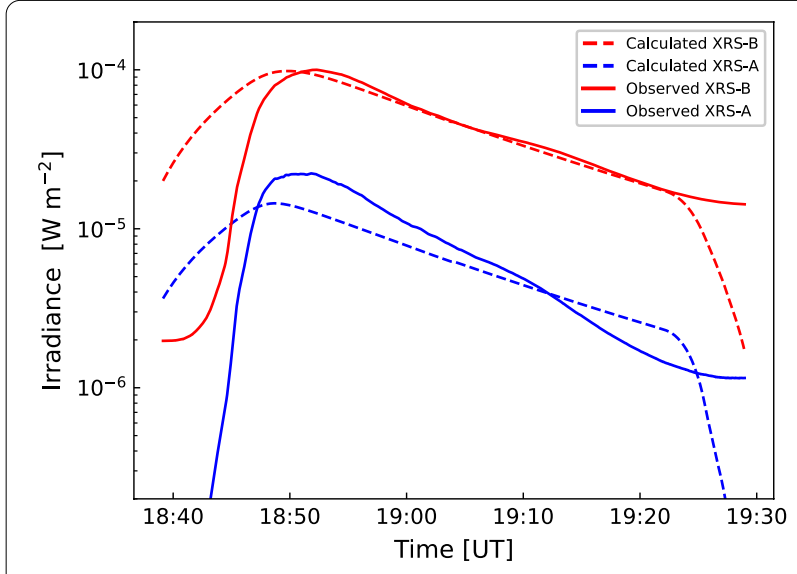

Fig. 7 Observed and reproduced light curves of GOES/XRS-A and GOES/XRS-B. The dashed and solid lines indicate the reproduced and observed light curves of GOES/XRS during the M9.9-class flare on January 1, 2014, respectively. The red and blue lines represent GOES/ XRS-B and GOES/XRS-A, respectively

Finally, we converted the synthetic flare emissions into a light curve that was comparable to the observation (see Section 3.5, Kawai et al. 2020). Figure 7 displays the reproduced GOES/XRS-B light curve obtained using the proposed model. We performed the conversion using the fit GOES/XRS-B light curve as described above. First, we calculated the ratio between the GOES/XRS-B observation and simulated GOES/XRS-B light curves (simply integrated calculated values of 1-8 $\AA$ ) for each observation time. Afterwards, we performed a convolution on the simulated GOES/XRS-B light curve with the observation. Subsequently, we calculated the ratio between the maximum value of the GOES/XRS-B observation and the maximum value of the convoluted GOES/XRS-B light curve, and we applied these two ratios to all simulated light curves. Finally, we derived the EUV emission spectra at all wavelengths that were comparable to the observations [see Figure 1 in Kawai et al. (2020)].

As the flare evolves, the reconnection point rises, the footpoints separate farther apart, and the flaring loop becomes longer. These geometric evolutions of flare affect the flare emission. Therefore, previous studies have reported that flare emission is well reproduced through the time evolution of multiple flare loops [e.g., Hori et al. (1997); Reep and Toriumi (2017)]. Our proposed model of convolving a simple calculation for a single loop into the GOES X-ray observation can 
roughly reproduce pseudoemissions from multiple flare loops as well as reduce computational complexity and cost.

Figure 8 displays an example of the EUV flare timeintegrated spectra during the M9.9-class flare on January 1,2014 . The solid red, blue, and green lines indicate the values obtained from the proposed model, FISM, and SDO/MEGS-A observations, respectively. The wavelength resolution was set to $10 \AA$. As indicated in Fig. 8, both the proposed model and FISM reproduced the tendencies of the observed values. Figure 9 displays the light curves of the EUV flare lines observed by SDO/EVE MEGS-A (top panel), estimated by the proposed model (middle panel), and the FISM (bottom panel) for the M9.9-class flare on January 1, 2014. The wavelength resolution of these light curves was $10 \AA$, which is the same as that of FISM. Therefore, the wavelengths of Fe VIII and Fe XX were $130 \AA$, Fe XV was $280 \AA$, Fe XVI was $330 \AA$, Fe XVIII was $90 \AA$, and He II was $300 \AA$, as indicated in Fig. 9. It is clear that the proposed model can reproduce the flux of each line based on the formation temperature; however, the He II emission line time series was not modeled correctly. Conversely, FISM provided a better representation of the timing of the He II emission line. The proposed model and FISM did not reproduce the secondary rise in Fe XV and Fe XVI at 19:20 UT. Table 1 presents the EUV flare time-integrated irradiance and EUV flare line rise time, observed by SDO/EVE MEGS-A and calculated by the proposed model for the M9.9-class flare on January $1,2014$.

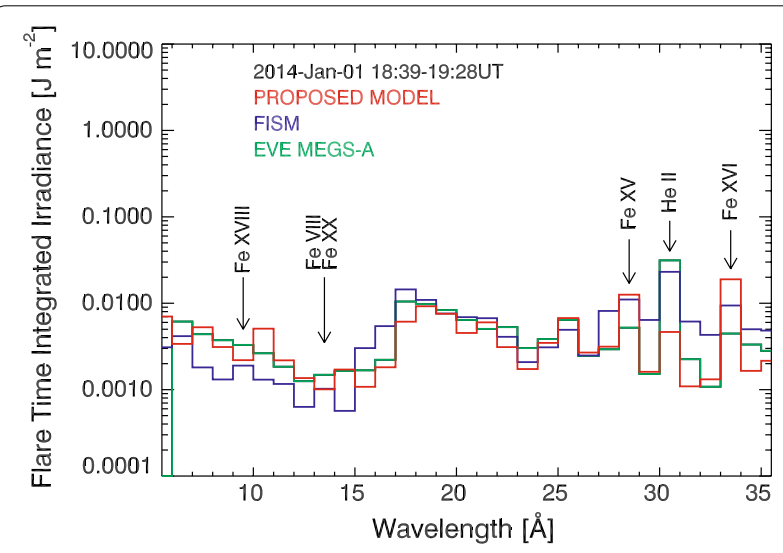

Fig. 8 Example of EUV time-integrated spectra during the flare. The EUV time-integrated spectra during the flare for the M9.9-class flare on January 1, 2014. The solid red, blue, and green lines indicate the calculated spectra of the proposed model, FISM, and observed spectra by SDO/EVE MEGS-A, respectively. The wavelength resolution is $10 \AA$. The arrows indicate the EUV lines focused on in this study

\section{Statistical study for comparison between model and observation}

We compared the EUV flare time-integrated irradiance and EUV flare line rise time obtained from the proposed model and SDO/EVE MEGS-A observations for 21 flare events using the same method as the event analysis in Sect. 4.

For the parameters of the proposed model used in this study, we determined $L$ from the observation of the ribbon distance described in Sect. 4.2, $H_{\mathrm{fo}}$ was selected such that the magnitude of the computed GOES/XRS light curve is scaled to best match the observation, and $w_{\mathrm{f}}$ and $\tau_{\mathrm{f}}$ are from the default parameter values of CANS 1D (as mentioned in Sect. 4.3). The GOES classes, flare dates, times, half-loop length estimations from the SDO/AIA observation, and the input energy used in the proposed model for 21 flare events are presented in Table 2.

First, we compared the flare time-integrated irradiance of each EUV line. Figure 10 displays the correlation of the EUV flare time-integrated irradiance of the six flare lines. All Fe lines had an acceptable correlation with the observations. However, the He II indicates poor correlation with a correlation coefficient of 0.24 (Fig. 10f). As indicated by the regression lines in Fig. 10, the Fe XVIII (Fig. 10d) effectively reproduced the observed data. Fe XV (Fig. 10b) and Fe XVI (Fig. 10c) tended to be overestimated; the Fe VIII (Fig. 10a), Fe XX (Fig. 10e), and He II (Fig. 10f) lines tended to be underestimated by the proposed model. Afterward, we compared the flare energies for two wavelength bands.

Figure 11 displays the comparison results of the EUV flare time-integrated irradiance of two wavelength bands of 55-355 $\AA$ (Fig. 11a) and 55-135 $\AA$ (Fig. 11b). The results of the proposed model and FISM are shown in red and blue, respectively. As indicated in Fig. 11a, the 55-355 ^ band was well reproduced by both the proposed model and FISM. The slopes of the regression lines were $0.81 \pm 0.08$ and $1.22 \pm 0.02$, respectively, and the correlation coefficients were 0.92 and 0.99 , respectively. Moreover, for the 55-135 $\AA$ band, the proposed model could reproduce the observations better than FISM (Fig. 11b). From Fig. 11b, the slope of the regression line for the proposed model was $0.91 \pm 0.08$, and the correlation coefficient was 0.94 . Conversely, the slope of the regression line for the FISM was $0.53 \pm 0.01$, and the correlation coefficient was 0.99 . These results indicate that the FISM tended to underestimate the EUV flare timeintegrated irradiance at this relatively shorter wavelength band.

Figure 12 displays the comparison results of the EUV flare line rise time. The correlation coefficient of the $\mathrm{Fe}$ VIII was 0.95 (Fig. 12a), Fe XV was 0.86 (Fig. 12b), Fe XVI was 0.90 (Fig. 12c), Fe XVIII was 0.92 (Fig. 12d), Fe XX 


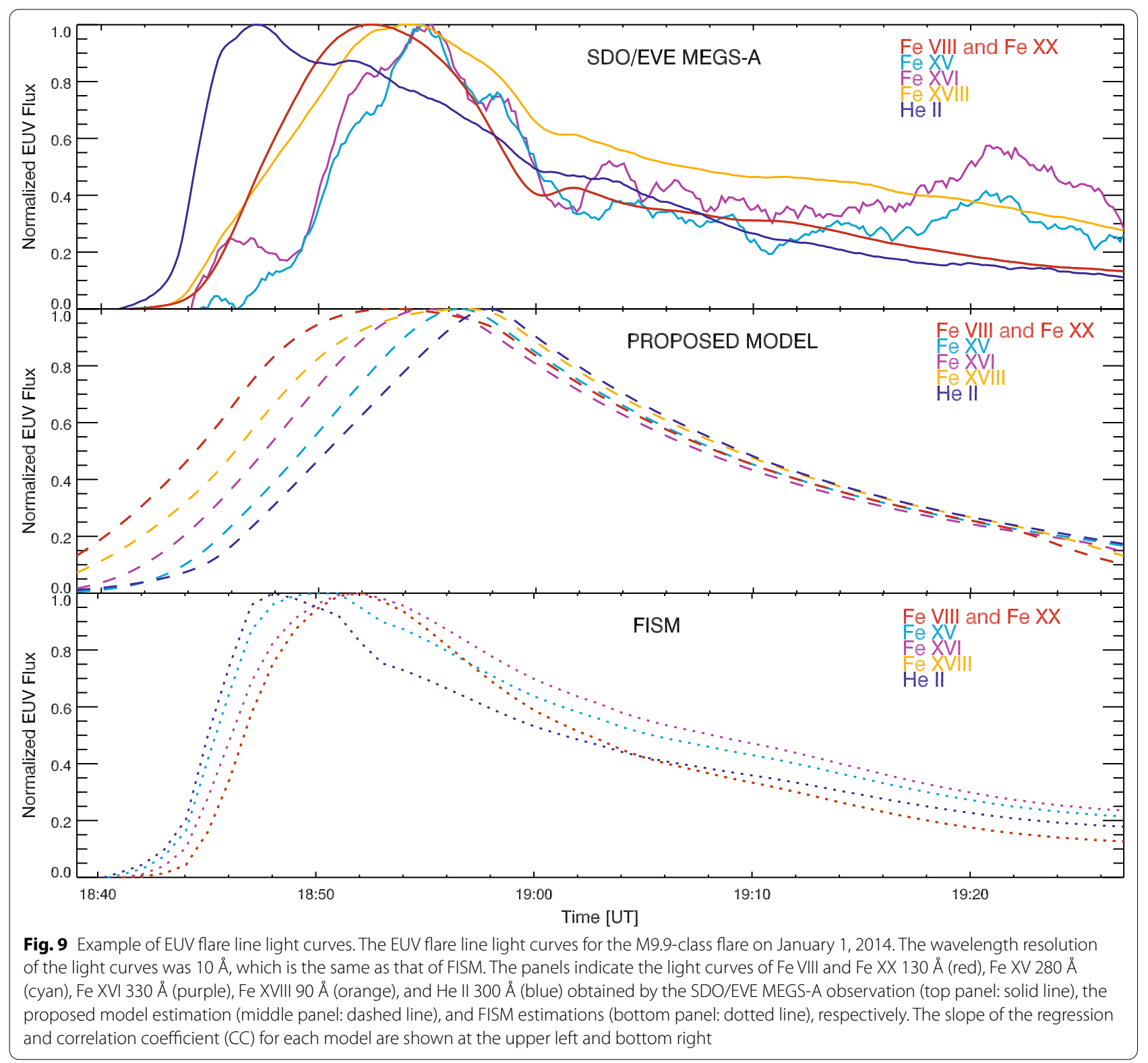

Table 1 Comparative parameters for the M9.9-class flare on January 1, 2014

\begin{tabular}{|c|c|c|c|c|}
\hline EUV flare line/band & $\begin{array}{l}\text { Observed EUV flare time-integrated } \\
\text { irradiance }\left(\mathrm{J} \mathrm{m}^{-2}\right)\end{array}$ & $\begin{array}{l}\text { Calculated EUV flare time- } \\
\text { integrated irradiance }\left(\mathrm{J} \mathrm{m}^{-2}\right)\end{array}$ & $\begin{array}{l}\text { Observed EUV flare line } \\
\text { rise time (s) }\end{array}$ & $\begin{array}{l}\text { Calculated EUV } \\
\text { flare line rise } \\
\text { time (s) }\end{array}$ \\
\hline Fe VIII & $1.3 \mathrm{E}-03$ & $7.9 \mathrm{E}-04$ & 939 & 988 \\
\hline Fe XV & $3.1 \mathrm{E}-02$ & $1.0 \mathrm{E}-01$ & 919 & 1056 \\
\hline Fe XVI & $2.6 \mathrm{E}-02$ & $1.7 \mathrm{E}-01$ & 949 & 956 \\
\hline Fe XVIII & 4.7E-03 & $3.7 \mathrm{E}-03$ & 919 & 1120 \\
\hline Fe XX & $3.0 \mathrm{E}-03$ & $2.8 \mathrm{E}-03$ & 779 & 648 \\
\hline Hell & $2.4 \mathrm{E}-01$ & $3.8 \mathrm{E}-02$ & 469 & 1144 \\
\hline $55-355 \AA$ & $1.4 \mathrm{E}-01$ & $1.3 \mathrm{E}-01$ & - & - \\
\hline $55-135 \AA$ & $2.4 \mathrm{E}-02$ & $2.4 \mathrm{E}-02$ & - & - \\
\hline
\end{tabular}


Table 2 Flare event list for this study

\begin{tabular}{|c|c|c|c|c|c|c|c|}
\hline Event no. & Date & GOES class & Flare start time (UT) & Flare peak time (UT) & Flare end time (UT) & $\begin{array}{l}\text { Estimated half- } \\
\text { loop length }(\mathrm{Mm})\end{array}$ & $\begin{array}{l}\text { Input energy } \\
\text { (erg cm}{ }^{-3} \mathrm{~s}^{-1} \text { ] }\end{array}$ \\
\hline 1 & 2011-07-30 & M9.3 & 02:04:33 & 02:09:38 & $02: 29: 36$ & 8.0 & 9.0 \\
\hline 2 & 2011-08-03 & M6.0 & 13:08:19 & 13:48:07 & $14: 51: 16$ & 39.6 & 3.0 \\
\hline 3 & 2011-09-24 & M3.1 & $17: 19: 12$ & $17: 25: 29$ & $17: 49: 35$ & 10.5 & 1.5 \\
\hline 4 & 2011-10-02 & M3.9 & $00: 31: 38$ & $00: 50: 24$ & $01: 24: 32$ & 17.5 & 3.0 \\
\hline 5 & 2011-11-05 & M3.7 & $02: 47: 20$ & 03:35:20 & 04:59:30 & 53.7 & 1.2 \\
\hline 6 & 2012-03-09 & M6.3 & $03: 22: 43$ & 03:49:53 & 04:53:57 & 38.0 & 3.0 \\
\hline 7 & 2012-03-10 & M8.4 & $17: 15: 15$ & $17: 27: 42$ & 19:09:46 & 13.7 & 6.0 \\
\hline 8 & 2012-05-09 & M4.7 & 12:19:04 & $12: 32: 11$ & 12:44:51 & 7.2 & 6.0 \\
\hline 9 & 2012-05-09 & M4.1 & 21:01:31 & $21: 05: 22$ & $21: 27: 48$ & 8.4 & 6.0 \\
\hline 10 & 2012-06-03 & M3.3 & $17: 48: 28$ & $17: 55: 13$ & 18:09:31 & 3.4 & 90.0 \\
\hline 11 & 2012-07-04 & M5.3 & 09:46:03 & 09:55:22 & 10:09:30 & 5.1 & 15.0 \\
\hline 12 & 2012-07-05 & M6.1 & 11:39:45 & $11: 44: 31$ & 12:19:31 & 4.9 & 15.0 \\
\hline 13 & 2013-04-12 & M3.3 & $20: 23: 50$ & 20:38:00 & $21: 14: 48$ & 16.5 & 24.0 \\
\hline 14 & 2013-10-28 & M4.4 & 15:06:10 & $15: 15: 44$ & 15:29:55 & 12.8 & 3.0 \\
\hline 15 & 2013-11-01 & M6.3 & 19:46:39 & 19:53:58 & $20: 19: 40$ & 8.9 & 4.5 \\
\hline 16 & 2013-11-05 & $\times 3.3$ & 22:05:56 & 22:12:56 & 22:38:08 & 12.5 & 12.0 \\
\hline 17 & 2013-11-06 & M3.8 & 13:35:29 & 13:49:02 & 14:08:07 & 7.0 & 6.0 \\
\hline 18 & 2013-11-08 & $X 1.1$ & 04:19:14 & 04:25:59 & $04: 49: 37$ & 11.4 & 6.0 \\
\hline 19 & 2013-11-10 & $X 1.1$ & 05:08:11 & $05: 14: 26$ & 05:49:32 & 3.7 & 300.0 \\
\hline 20 & 2014-01-01 & M9.9 & 18:39:14 & 18:52:12 & 19:29:39 & 13.3 & 6.0 \\
\hline 21 & 2014-02-12 & M3.7 & $04: 14: 47$ & $04: 25: 28$ & 04:53:56 & 12.0 & 3.0 \\
\hline
\end{tabular}

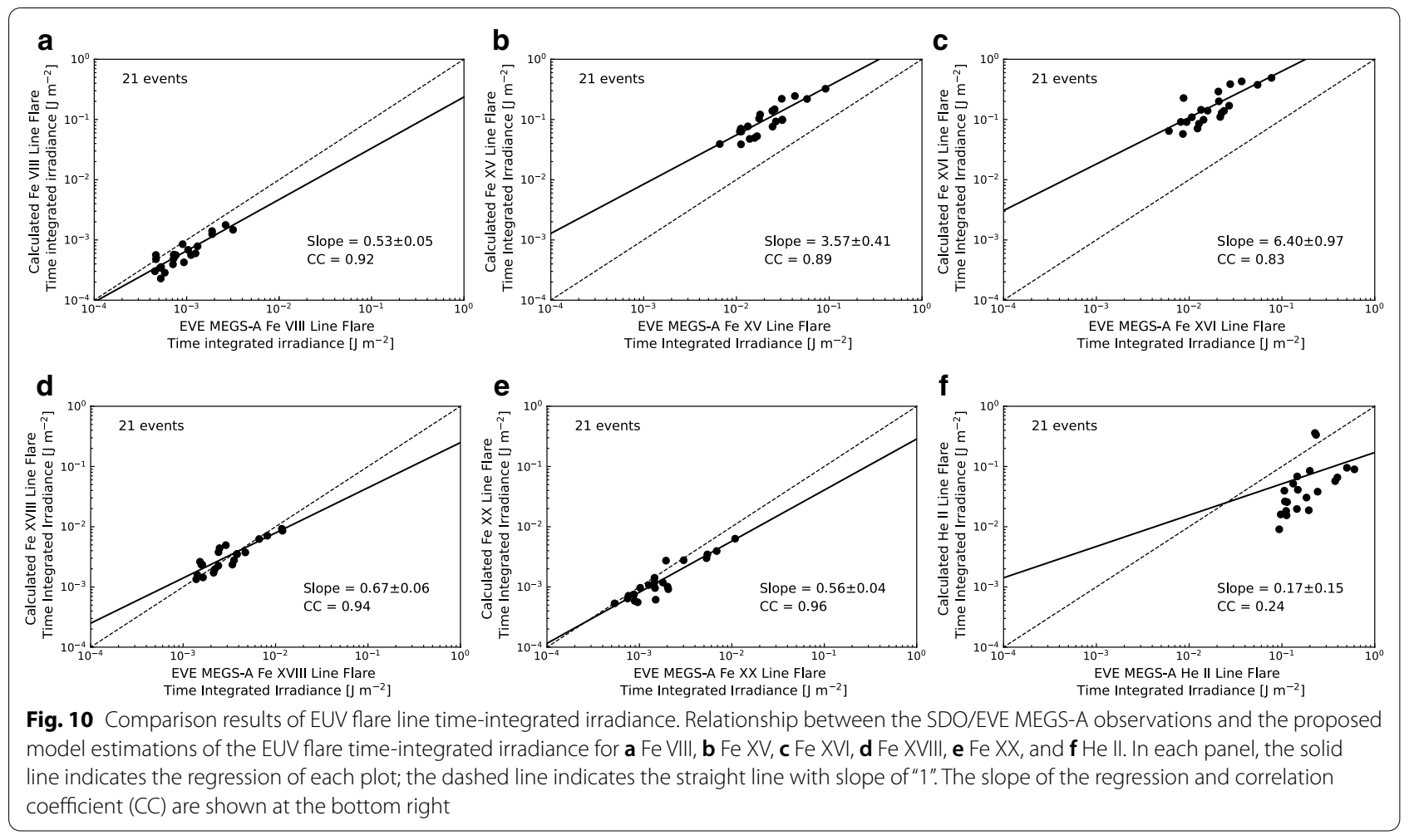



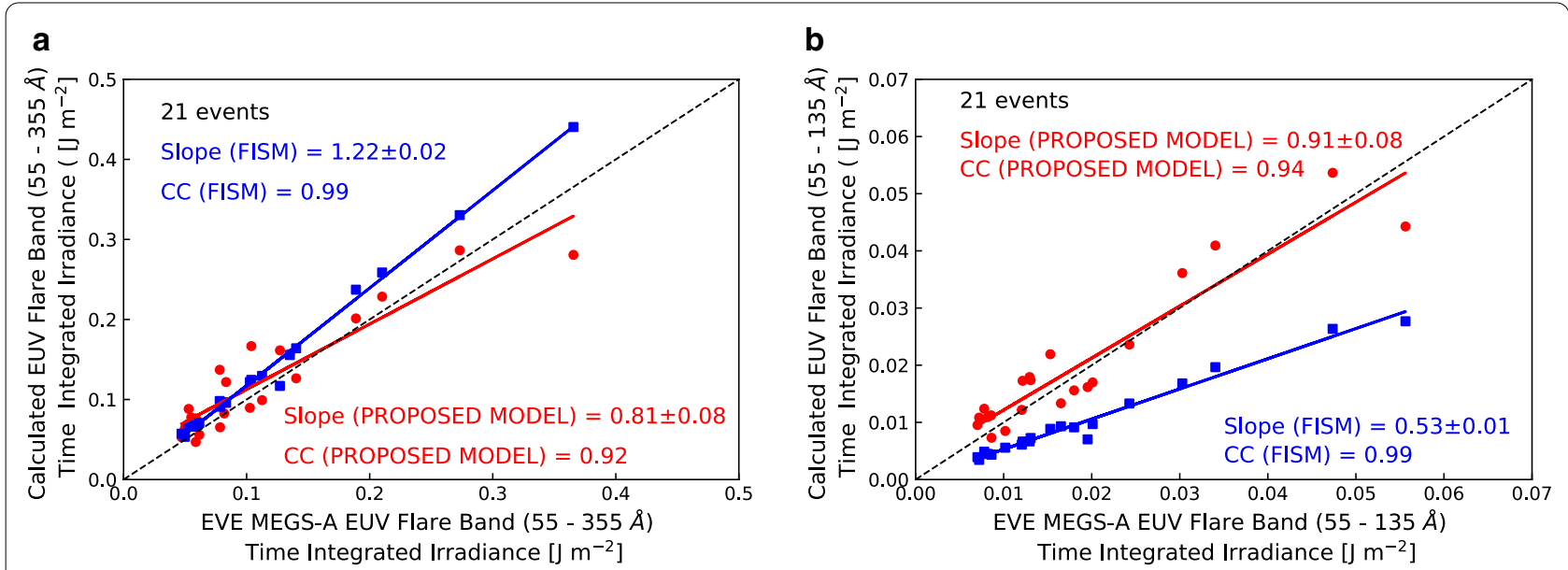

Fig. 11 Comparison results of EUV flare band time-integrated irradiance. The relationship between the SDO/EVE MEGS-A observations and proposed model estimations of the EUV flare energies of two wavelength bands for $\mathbf{a} 55-355 \AA$ and $\mathbf{b}$ 55-135 $\AA$. The results of the proposed model are plotted in red, and the FISM results are plotted in blue. In each panel, the solid line indicates the regression of each plot; the dashed line indicates the straight line with slope of "1" and the slope of regression is shown at the upper left and bottom right
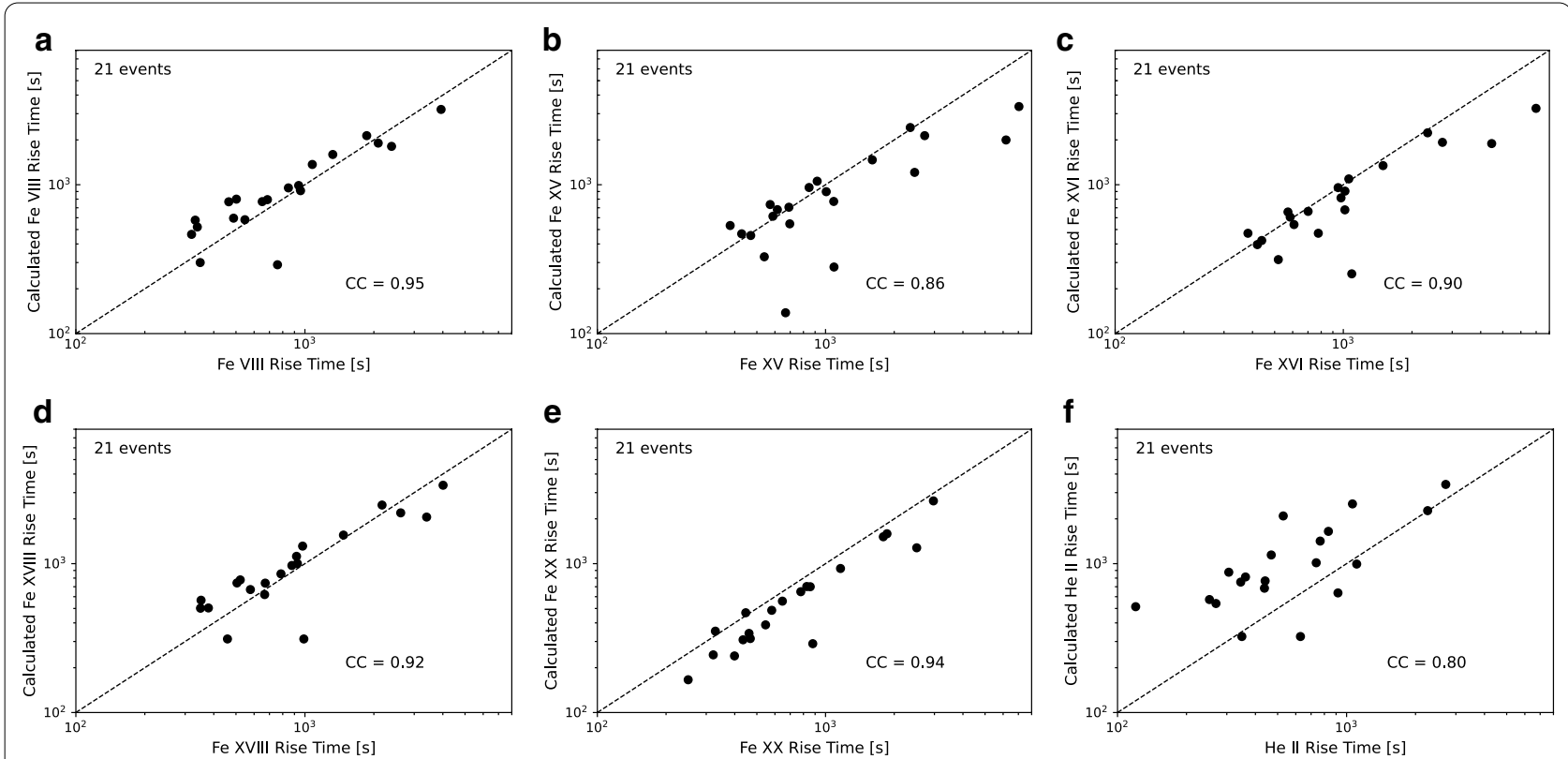

Fig. 12 Comparison results of the EUV flare line rise time. The relationship between the SDO/EVE MEGS-A observations and proposed model estimations of the EUV flare line rise time for a Fe VIII, b Fe XV, c Fe XVI, d Fe XVIII, e Fe XX, and $\mathbf{f} H$ He II. In each panel, the dashed line indicates the straight line with slope of " 1 " and the correlation coefficient (CC) is shown at the bottom right

was 0.94 (Fig. 12e), and He II was 0.80 (Fig. 12f). It can be clearly observed that all Fe lines indicated acceptable correlations with the observations. From these scatter plots, the calculated rise times of Fe XV, Fe XVI, and Fe $\mathrm{XX}$ tended to be less than the observations (Fig. 12b, c, e), whereas that of He II tended to be greater than the observations (Fig. 12f).

\section{Discussion and summary}

We attempted to reproduce EUV flare emission spectra using a simple physics-based model that simulates the time evolution of the emitting plasma distribution in the flare loop. We verified the EUV flare time-integrated irradiance and EUV flare line rise time of EUV line emissions for 21 events by comparing the calculation results of the proposed 
model and observed EUV spectral data. In this study, we used FISM to compare with the proposed model; it should be noted that the FISM2 was released quite recently (Chamberlin et al. 2020). Some of the most significant accuracy improvements in FISM2 are in the 60-270 $\AA$ range, an important region discussed and compared throughout this paper. Future analysis must be performed using FISM2.

For the flare time-integrated irradiance, the proposed model succeeded in reproducing the Fe VIII, Fe XVIII, and Fe XX lines (Fig. 10a, d, e) as well as the 55-355 and 55-135 $\AA$ bands (Fig. 11a). Our results indicate that the physics-based model was effective in reproducing EUV flare irradiance for wavelengths shorter than $140 \AA$. Conversely, the proposed model overestimated the relatively longer wavelength lines, Fe XV and Fe XVI lines, emitted from relatively cooler plasmas (Fig. 10b, c). Thiemann et al. (2018) reported that the peak EUV irradiance of emission lines with higher formation temperatures (>9 MK) is proportional to the GOES/XRS-B peak emission measure. Therefore, our results imply that the EUV irradiance of the emitting plasmas with a lower formation temperature is not linearly proportional to the irradiance of the hotter emission lines or soft X-ray flux.

Regarding the time evolution of the EUV flare line emission, the proposed model could derive the time evolution of the EUV line emission by simulating the time evolution of the temperature in the flare loop. This tendency can be observed because the difference in peak time depending on the formation temperature of the emitting plasma was reproduced by the proposed model, as indicated in Fig. 9. The Fe XX line, which was emitted from the highest formation temperature plasma, had the shortest rise time (Fig. 12d), and the He II line, which emitted from the lowest formation temperature, had the longest rise time in the proposed model (Fig. 12f). This is because the time evolution of each EUV flare line is determined by the temperature of the emitting plasma in the proposed model. The proposed model and FISM did not reproduce the secondary rise in Fe XV and Fe XVI at $\sim$ 19:20 UT, as shown in Fig. 9. The characteristic of the EUV line emission seen in Fe XV and Fe XVI is known as the EUV late phase and is considered to be due to the second set of post-flare loops (Woods et al. 2011). The EUV late phase of Fe XV and Fe XVI is a feature that does not appear in GOES/XRS-B observation; therefore, we consider it difficult to reproduce with FISM or the proposed model. It is necessary to reproduce the physical process of flare in more detail to reproduce the time evolution of such characteristic flare emissions.

For the He II emission line, both the time-integrated irradiance (Fig. 10f) and EUV flare line rise time (Fig. 12f) were poorly reproduced by the proposed model. This is because the proposed model neglected the EUV emissions from below the transition region, which is the origin of the optically thick plasmas; hence, we did not calculate the radiation transfer. The proposed model is primarily based on the physical processes following soft $\mathrm{X}$-ray emission. Therefore, future work includes the construction of a model to accurately reproduce the EUV emission from optically thick plasmas dominant in the impulsive phase.

In this study, for the most part, we used the default parameter values set in CANS 1D (see the CANS 1D documentation). Then, we used the half-loop length estimated from the observation and set the optimal input energy for each flare event as a variable parameter. The parameters used are listed in Table 2. Our results indicate that the EUV emissions from emitting plasma with a relatively high formation temperature can be reproduced using only the flare input energy as a variable parameter.

As mentioned above, our results indicate that the physics-based model is effective for reproducing EUV flare emissions. The proposed model successfully reproduced the EUV emission intensity of Fe lines with a high formation temperature and the time evolution of all Fe lines during a flare. Since the Earth's ionosphere and thermosphere are sensitive to EUV emissions from the Sun (Qian et al. 2011), an accurate estimation of the EUV irradiance and duration during solar flares is vital when considering space weather. Therefore, we consider that the simple model used in this study can contribute to the space weather forecast operations.

\section{Abbreviations \\ AIA: Atmospheric Imaging Assembly; CANS: Coordinated Astronomical Numerical Software; CC: Correlation coefficient; CCD: Charge-coupled device; EBTEL: Enthalpy-based Thermal Evolution of Loops; EUV: Extreme ultraviolet; EVE: Extreme ultraviolet variability experiment; FISM: Flare Emission Spectral Model; GOES: Geostationary Operational Environmental Satellite; MEGS: Multiple EUV Grating Spectrograph; SEE: Solar EUV Experiment; SEM: Solar EUV Monitor; SDO: Solar Dynamics Observatory; SIDs: Sudden ionospheric disturbances; SOHO: Solar and Heliospheric Observatory; TIMED: Thermo- sphere lonosphere Mesosphere Energetics and Dynamics; UV: Ultraviolet; UT: Universal time; XRS: X-ray sensors.}

\section{Acknowledgements}

The authors would like to thank to K. Ichimoto, S. Takasao, S. Toriumi, H. P. Warren, and J. W. Reep for stimulating fruitful discussions.

\section{Authors' contributions}

SN performed statistical study for solar flare spectrum data and drafted the manuscript. KW, TK, SI and TK discussed the results and edited the manuscript. All the authors read and approved the final manuscript.

\section{Funding}

Part of this work was conducted by the joint research program of the Institute for Space-Earth Environmental Research (ISEE), Nagoya University. This

study was supported by JSPS KAKENHI Grant Numbers JP16H01187, and JP18H04452.

Availability of data and materials

The SDO/EVE level 2 data version 6 data are available at http://lasp.colorado. edu/eve/data_access/eve_data/products/level2/. FISM data are available at 
https://lasp.colorado.edu/lisird/data/fism_flare_hr/. The flare event data used in this study can be found in Hinode flare catalogue (https://hinode.isee. nagoya-u.ac.jp/flare_catalogue/).

\section{Declarations}

\section{Ethics approval and consent to participate}

Not applicable.

\section{Consent for publication}

Not applicable.

\section{Competing interests}

The authors declare that they have no competing interests.

\section{Author details}

${ }^{1}$ National Defense Academy of Japan, 1-10-20 Hashirimizu, Yokosuka 239-8686, Japan. ${ }^{2}$ Institute for Space-Earth Environmental Research (ISEE), Nagoya University, Furo-cho, Chikusa-ku, Nagoya 464-8601, Japan.

${ }^{3}$ National Institute for Fusion Science, 322-6 Oroshi-cho, Toki 509-5292, Japan.

Received: 5 August 2020 Accepted: 18 March 2021

Published online: 25 March 2021

\section{References}

Asai A, Ishii TT, Kurokawa H, Yokoyama H, Shimojo M (2003) Evolution of conjugate footpoints inside flare ribbons during a great two-ribbon flare on 2001 April 10. Astrophys J 586:624-629

Bornmann PL, Speich D, Hirman J et al (1996) GOES X-ray sensor and its use in predicting solar-terrestrial disturbances. Proc SPIE 2812:291-298

Cargill PJ, Bradshaw SJ, Klimchuk JA (2012) Enthalpy-based thermal evolution of loops. III. Comparison of zero-dimensional models. Astrophys J 758:5

Carmichael H (1964) A process for flares. Proc AAS-NASA Symp 50:451

Chamberlin PC, Woods TN, Eparvier FG (2006) Flare emission spectral model (FISM) use for space weather applications. In: Proceedings of the ILWS workshop, p 153

Chamberlin PC, Woods TN, Eparvier FG (2007) Flare emission spectral model (FISM): daily component algorithms and results. Space Weather 5:S07005. https://doi.org/10.1029/2007SW000316

Chamberlin PC, Woods TN, Eparvier FG (2008) Flare emission spectral model (FISM): flare component algorithms and results. Space Weather 6:S05001. https://doi.org/10.1029/2007SW000372

Chamberlin PC, Eparvier FG, KnoerV et al (2020) The flare irradiance spectral model-version 2 (FISM2). Space Weather 18:SW002588. https://doi.org/10. 1029/2020SW002588

Dellinger JH (1937) Sudden disturbances of the ionosphere. J Appl Phys 8:732-751

Dere KP, Landi E, Mason HE, Monsingnori BC, Young PR (1997) CHIANTI—an atomic database for emission lines. Astron Astrophys Suppl 125:149-173

Dere KP, Zanna GD, Young PR, Landi E, Sutherland RS (2019) CHIANTI —an atomic database for emission lines. XV. Version 9, improvements for the X-ray satellite lines. Astrophys I Suppl 241:22

Eparvier FG, Crotser D, Jones AR et al (2009) The extreme ultraviolet sensor (EUVS) for GOES-R. Sol Phys Space Weather Instrum III. https://doi.org/10.1117/12. 826445

Hirayama T (1974) Theoretical model of flares and prominences. I: evaporating flare model. Sol Phys 34:323-338

Hori K, Yokoyama T, Kosugi T, Shibata K (1997) Pseudo-two-dimensional hydrodynamic modeling of solar flare loops. Astrophys J 489:426-441

Imada S, Zweibel EG (2012) Self-organization of reconnecting plasma to marginal collisionality in the solar corona. Astrophys J 755:93

Imada S, Aoki K, Hara H, Watanabe T, Harra LK, Shimizu T (2013) Evidence for hot fast flow above a solar flare arcade. Astrophys J Lett 776:L11

Imada S, Murakami I, Watanabe T (2015) Observation and numerical modeling of chromospheric evaporation during the impulsive phase of a solar flare. Phys Plasmas 22:101206. https://doi.org/10.1063/1.4932335

Innes DE, McKenzie DE, Wang TJ (2003) Observations of 1000 km Doppler shifts in $107 \mathrm{~K}$ solar flare supra-arcade. Sol Phys 217:267-279
Judge DL, McMullin DR, Ogawa HS et al (1998) First solar EUV irradiances obtained from SOHO by the CELIAS/SEM. Sol Phys 177:161-173

Kawai T, Imada S, Nishimoto S, Watanabe K, Kawate T (2020) Nowcast of an EUV dynamic spectrum during solar flares. J Atmos Sol Terr Phys 205:105302

Klimchuk JA, Patsourakos S, Cargill PJ (2008) Highly efficient modeling of dynamic coronal loops. Astrophys J 682:1351-1362

Kopp RA, Pneuman GW (1976) Magnetic reconnection in the corona and the loop prominence phenomenon. Sol Phys 50:85-98

Lee KS, Imada S, Watanabe K, Bamba Y, Brooks DH (2017) IRIS, Hinode, SDO, and RHESSI observations of a white light flare produced directly by nonthermal electrons. Astrophys J 836:150

Lemen JR, Title AM, Boerner PF et al (2012) The atmospheric imaging assembly (AIA) on the solar dynamics observatory (SDO). Sol Phys 275:17-40

LiY, Qiu J, Ding M (2014) Heating and dynamics of two flare loop systems observed by AIA and EIS. Astrophys J 781:120

Milligan RO, Dennis BR (2009) Velocity characteristics of evaporated plasma using Hinode/EUV imaging spectrometer. Astrophys J 699:968-975

Qian L, Burns AG, Chamberlin PC, Solomon SC (2011) Variability of thermosphere and ionosphere responses to solar flares. J Geophys Res Space Phys 116:A10309. https://doi.org/10.1029/2011JA016777

Reep JW, Toriumi S (2017) The direct relation between the duration of magnetic reconnection and the evolution of GOES light curves in solar flares. Astrophys J 851:4

Shiota D, Isobe H, Chen PF, Yamamoto T, Sakajiri T, Shibata K (2005) Self-consistent magnetohydrodynamic modeling of a coronal mass ejection, coronal dimming, and a giant cusp-shaped arcade formation. Astrophys J 634:663-678

Spitzer L (1962) Physics of fully ionized gases. Interscience Publishers, New York

Strickland DJ, Evans FS, Paxton $\amalg$ (1995) Satellite remote sensing of thermospheric O/ $\mathrm{N}_{2}$ and solar EUV: 1. Theory. J Geophys Res 100:12217-12226

Sturrock PA (1966) Model of the high-energy phase of solar flares. Nature 211:695-697

Temmer M, Veronig AM, Vršnak B, Miklenic C (2007) Energy release rates along Ha flare ribbons and the location of hard X-ray sources. Astrophys J 654:665-674

Thiemann EMB, Eparvier FG, Woods TN (2017) A time dependent relation between EUV solar flare light-curves from lines with differing formation temperatures. J Space Weather Space Clim 7:A36. https://doi.org/10.1051/ swsc/2017037

Thiemann EMB, Chamberlin PC, Eparvier FG, Epp L (2018) Center-to-limb variability of hot coronal EUV emissions during solar flares. Sol Phys 293:19

Thiemann EMB, Eparvier FG, Woodraska D et al (2019) The GOES-R EUVS model for EUV irradiance variability. J Space Weather Space Clim 9:A43. https://doi. org/10.1051/swsc/2019041

Warren HP, Brooks DH, Ugarte-Urra l et al (2018) Spectroscopic observations of current sheet formation and evolution. Astrophys J 854:122

Watanabe K, Masuda S, Segawa T (2012) Hinode flare catalogue. Sol Phys 279:317-322

Woods TN, Eparvier FG, Bailey SM et al (2005) Solar EUV experiment (SEE): mission overview and first results. J Geophys Res Space Phys 110:A01312. https:// doi.org/10.1029/2004JA010765

Woods TN, Hock R, Eparvier FG et al (2011) New solar extreme-ultraviolet irradiance observations during flares. Astrophys $J$ 739:59

Woods TN, Eparvier FG, Hock R et al (2012) Extreme ultraviolet variability experiment (EVE) on the solar dynamics observatory (SDO): overview of science objectives, instrument design, data products, and model developments. Sol Phys 275:115-143

Yokoyama T, Shibata K (1998) A two-dimensional magnetohydrodynamic simulation of chromospheric evaporation in a solar flare based on a magnetic reconnection model. Astrophys J 494:L113-L116

Zeng Z, Qiu J, Cao W, Judge PG (2014) A flare observed in coronal, transition region, and helium I 10830 Å emissions. Astrophys J 793:87

\section{Publisher's Note}

Springer Nature remains neutral with regard to jurisdictional claims in published maps and institutional affiliations. 